\title{
PRRT2 Gene Analysis of Paroxysmal Kinesigenic Dyskinesia (PKD) in Thai Children
}

\author{
Pantaree Laosuebsakulthai, M.D. ${ }^{1}$, Surachai Likasitwattanakul, M.D. ${ }^{1}$, \\ Theerapong Pho-iam, Ph.D. ${ }^{2}$, Wanna Thongnoppakhun, Ph.D. ${ }^{2}$, \\ Mongkol Chanvanichtrakool, M.D. ${ }^{1}$
}

'Division of Neurology, Department of Pediatrics, Faculty of Medicine, Siriraj Hospital, Mahidol University, Bangkok Noi, Bangkok 10700, Thailand.

2Division of Medical Genetics Research and Laboratory, Research Department, Faculty of Medicine, Siriraj Hospital, Mahidol University, Bangkok Noi, Bangkok 10700, Thailand.

Received 17 August 2021 • Revised 3 October 2021 • Accepted 14 October 2021 • Published online 24 November 2021

\begin{abstract}
:
Objective: To examine the frequency of the proline-rich transmembrane protein-2 (PRRT2) gene mutation in Thai patients with paroxysmal kinesigenic dyskinesia (PKD).

Material and Methods: A retrospective study of children aged 0-18 years with a diagnosis of PKD at Siriraj Hospital. The genetic analyses of the PRRT2 gene were done by bidirectional Sanger sequencing.

Results: Twelve patients with PKD were included. The known PRRT2 mutation, c.649dupC (p.Arg217Profs*8), was identified in three of the patients (25.0\%), one of the nine sporadic cases (11.1\%) and two of the three familial cases $(66.6 \%)$, all from different families. PKD had a complete response to carbamazepine treatment regardless of PRRT2 mutation status. Conclusion: Our study provided the new details of the clinical phenotypes and PRRT2 gene analysis findings for Thai PKD. PRRT2 mutations were identified in our Thai PKD patients with increased detection rates in the familial PKD cases. The c.649dupC (p.Arg217Profs*8) was also found to be a hot-spot mutation in our Thai PKD patients. Furthermore, this study demonstrates the importance of PRRT2 gene analysis in order to properly diagnose and treat these patients.
\end{abstract}

Keywords: paroxysmal kinesigenic dyskinesia, PKD, PRRT2 gene, Thai children

Contact: Mongkol Chanvanichtrakool, M.D.

Division of Neurology, Department of Pediatrics, Faculty of Medicine, Siriraj Hospital, Mahidol University, Bangkok Noi, Bangkok 10700, Thailand.

E-mail: Mongkol.cha@mahidol.edu

(c) 2021 JHSMR. Hosting by Prince of Songkla University. All rights reserved.

This is an open access article under the CC BY-NC-ND license

(http://www.jhsmr.org/index.php/jhsmr/about/editorialPolicies\#openAccessPolicy).
J Health Sci Med Res 2022;40(4):419-424 doi: $10.31584 /$ jhsmr.2021853 www.jhsmr.org 


\section{Introduction}

Paroxysmal kinesigenic dyskinesia (PKD) is a rare, heterogenous group of movement disorders. The estimated prevalence is approximately 1 per $150,000 .^{1,2}$ PKD can be sporadic or familial in an autosomal dominant inheritance. Sporadic PKD was reported in $58.8-71.4 \%$ of the cases in two previous studies. ${ }^{3,4}$ The clinical presentation is characterized by brief involuntary movements triggered by a sudden or changed direction of movement. Such movements can be caused by dystonia, chorea, ballism, or a combination of these. The symptoms respond well to low-dose carbamazepine (CBZ) and tend to decrease with age. , $^{2,5}$

Mutations of the proline-rich transmembrane protein-2 (PRRT2) gene located on chromosome 16p11.2 have been identified as a cause of PKD. ${ }^{6}$ Various studies have reported that a PRRT2 mutation was found in 60.0$92.8 \%$ of the familial and $13.6-45.0 \%$ of the sporadic PKD cases. $^{3,4,7}$ Another study reported that $78.5 \%$ of the cases had a c.649dupC frameshift mutation. ${ }^{8}$ The clinical spectrum of PRRT2-related diseases involves benign familial infantile convulsions (BFIC), infantile convulsions with choreoathetosis (ICCA), hemiplegic migraine, and episodic ataxia. ${ }^{8}$ Apart from the PRRT2 mutations, other causative genes of PKD have been reported, such as $S L C 2 A 1^{1}, S C N 8 A^{9}, K C N M A 1, K C N A 1, D E P D C 5^{10}$ and a deletion of chromosome 16p11.2. ${ }^{11}$

A study of clinical phenotypes and genetic testing in Thai PKD patients has never been done. Our study aimed to assess the frequency of PRRT2 mutations and characterize the clinical features of PKD along with the diagnostic impact of PRRT2 gene mutations in Thai patients.

\section{Material and Methods}

This was a retrospective study which included children aged 0-18 years with a diagnosis of PKD who met Bruno's criteria ${ }^{6}$ between January, 2007 and May, 2019. The demographic data, clinical phenotypes, and results of genetic and neuroimaging studies and treatment outcomes were collected by chart review. The protocol for this study was approved by the Siriraj Institutional Review Board, Faculty of Medicine, Siriraj Hospital, Mahidol University, Bangkok, Thailand.

Genomic deoxyribonucleic acid (DNA) samples from peripheral blood were extracted using the standard saltingout procedure. A mutation analysis of the PRRT2 gene was carried out by Sanger sequencing. Then Polymerase Chain Reaction (PCR) with three newly designed primer pairs was performed to amplify the entire coding sequence of the PRRT2 gene. The PCR amplified fragments were treated with ExoSAP-IT (Applied Biosystems, USA) and subsequently sequenced in both directions using a BigDye Terminator v3.1 Cycle Sequencing kit (Applied Biosystems, USA) with the same primers used in the PCR reactions. The sequencing products were run on an Applied Biosystems 3130xl Genetic Analyzer (Applied Biosystems, Foster City, CA, USA). The obtained sequences were compared with the reference sequence (NM_145239.3). Sequencevariants identification was reported based on the Human Genome Variation Society nomenclature ${ }^{12}$ and the classified pathogenicity according to the American College of Medical Genetics and Genomics (ACMG) guidelines. ${ }^{13}$

The data were described by descriptive statistics. The continuous data were reported by mean and standard deviation in a normal distribution or a median and interquartile range in a non-normal distribution. Categorical data were reported by percentage.

\section{Results}

A total of 12 patients with a diagnosis of PKD during the study period were recruited. Of these, nine were male and three had a family history of first-degree relatives with PKD. One patient (in the familial PKD group) had a history of BFIC. The clinical characteristics, investigations, and treatment results are summarized in Table 1. 


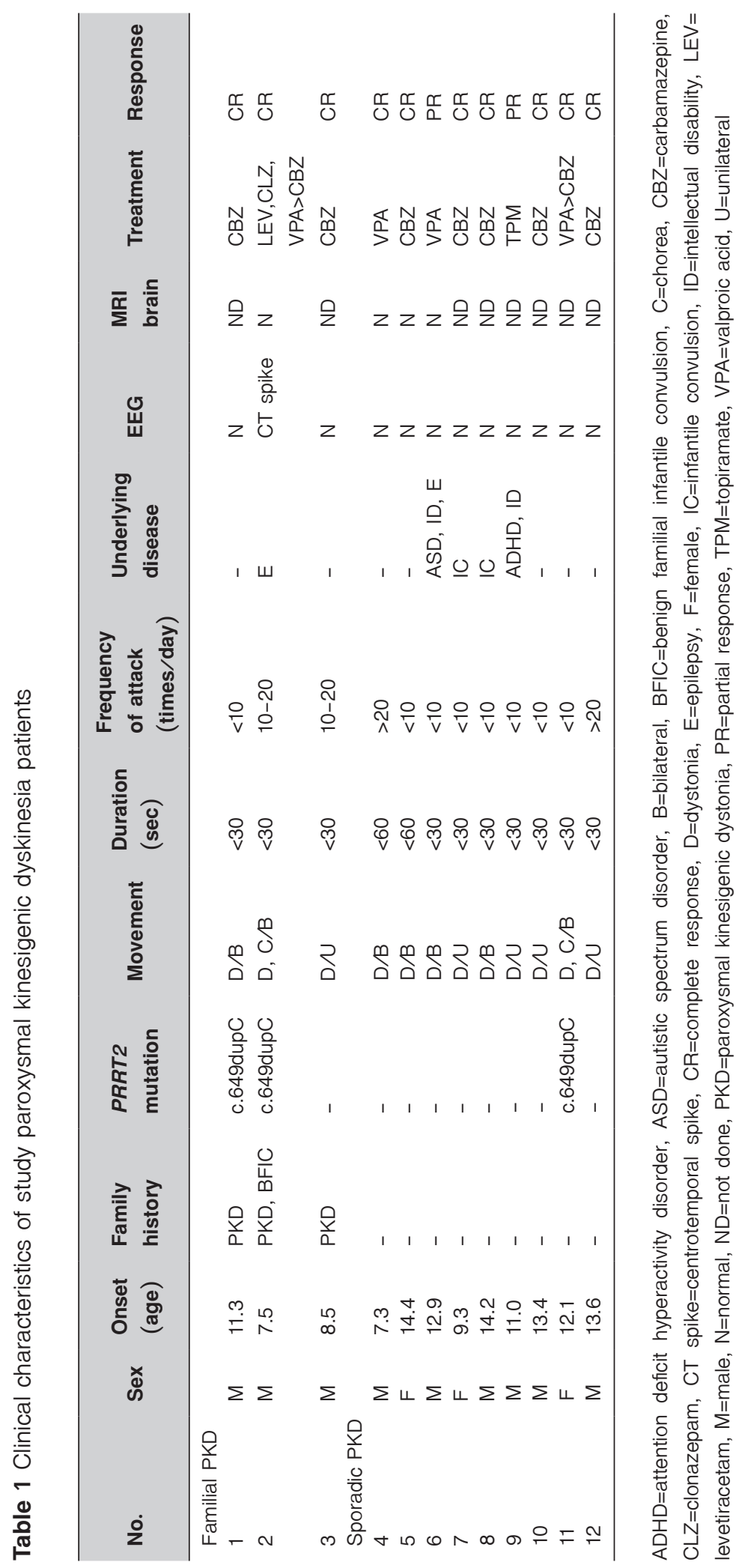




\section{Clinical characteristics}

The mean age of onset was $11.3 \pm 2.6$ years, and the median time to diagnosis was 1.1 (IQR, 0.2-1.5) years. All patients had dystonia triggered by the initiation of movement, and in three patients $(25.0 \%)$ the dystonia was triggered by excitement. Two of them also had chorea. Seven (58.3\%) patients had bilateral dyskinesia. Aurae such as general unease and/or tingling sensation were reported in nine patients (75.0\%). Two individuals had an intellectual disability.

\section{Investigation results}

Pathogenic PRRT2 mutations were identified in three patients: two of the three in the familial and one out of nine in the sporadic PKD groups. All mutations were of the heterozygous frameshift type of c.649dupC (p.Arg217Profs $\left.{ }^{\star} 8\right)$.

We performed electroencephalography (EEG) in all patients and their results were normal, except for one patient (no. 2), who had a bilateral centro-temporal spike. Four patients had magnetic resonance imaging of the brain, and all were normal.

\section{Treatment results}

Nine patients had a complete response to CBZ, with a mean dose of $3.8 \pm 3.0 \mathrm{mg} / \mathrm{kg} /$ day. Other antiseizure medications (ASMs) were given to patients who had a positive HLA-B*1502 screening test. Four patients received sodium valproate, and there was a complete response in one of them and more than a $50.0 \%$ reduction of attacks in three. The average dose of sodium valproate was $20.0 \pm$ $11.7 \mathrm{mg} / \mathrm{kg} /$ day. One patient received topiramate and had a partial response.

A misdiagnosis of PKD was made on patient no. 2. He had briefly developed focal to bilateral, generalized tonic-clonic seizures when he was five years old. His EEG showed a bilateral centrotemporal region with a bilateral, generalized 2-3 Hz spike-wave complex during sleep. His seizures were controlled with phenobarbital. At age seven, he started having paroxysmal events characterized by a brief focal tonic stiffening of the limbs, with preserved consciousness. Due to his previous diagnosis of epilepsy and abnormalities in his EEG, he had been misdiagnosed as epilepsy for two years after the new symptoms started. Multiple ASMs were given to him, sodium valproate, clonazepam, levetiracetam, and topiramate, but without effect. He was then transferred to Siriraj Hospital for further evaluation. The paroxysmal events were captured during a study of his EEG and revealed no ictal EEG activity. It was later determined that all of his paroxysms were triggered by the initiation of movement. PRRT2 gene sequencing was then obtained, and revealed heterozygous c.649dupC. Low-dose CBZ was given to him, and this resulted in rapid and complete treatment response.

\section{Discussion}

Our study reviewed the clinical phenotypes and the results of PRRT2 gene sequencing in Thai patients with PKD. Overall, the clinical phenotypes were similar to those reported in other studies. ${ }^{3,4,7}$ Male predominance was observed in our study. All of our patients typically presented with dystonia during late childhood or adolescence. Our treatment results showed that all PKD patients who received low-dose CBZ had a good response. This supports prior research projects which concluded that $\mathrm{CBZ}$ is the most effective medication for PKD patients. ${ }^{3}$ In patients with positive HLA-B*1502 screening, other ASMs such as sodium valproate or topiramate were used and led to either a complete or partial response.

PKD can be familial or sporadic. Most of our patients were sporadic PKD cases $(75.0 \%)$, which is similar to previous studies (58.8-71.4\%). ${ }^{3,4}$ PRRT2 mutations were reported in $60.0-92.8 \%$ of familial and $13.6-45.0 \%$ of sporadic cases. ${ }^{3,4,7}$ In our study, PRRT2 gene mutations 
were found in $3 / 12(25.0 \%)$ of all the patients: $2 / 3(66.7 \%)$ in the familial and $1 / 9(11.1 \%)$ in the sporadic cases. The ratio of PRRT2 mutations in familial PKD was higher than among the sporadic PKD cases. These findings correspond well with three Korean and one Chinese studies. ${ }^{7,14}$

More than 80 different PRRT2 mutations have been identified ${ }^{10}$, including truncating, missense, and splice-site mutations and a complete PRRT2 deletion. Of these, the c.649dupC frameshift mutation has been most commonly found $(80.5 \%)$ in PKD patients. ${ }^{8}$ The pathogenesis can be explained by the nature of the nucleotide sequence, in which nine consecutive cytosines $(C)$ are located at positions 649657 , thus facilitating the slippage of DNA polymerase during the DNA replication process, and leading to an insertion of one $\mathrm{C}$. This frameshift mutation introduces a premature stop codon which causes truncated PRRT2 proteins with a consequent loss of function. ${ }^{15,16}$ In our study, all mutations were of the c.649dupC (p.Arg217Profs ${ }^{\star} 8$ ) type. This finding corresponds with that of other studies - namely, that this site is a hot-spot mutation in Thai PKD.,8

Although the PRRT2 gene is the most common gene identified in patients diagnosed PKD, PRRT2 mutations have not been detected in up to $40.0 \%$ of the familial and most of the sporadic PKD cases. Therefore, other causative PKD genes could be found in patients with negative PRRT2 mutations including $S L C 2 A 1^{1}, S C N 8 A^{9}$, KCNMA1, KCNA1, $D E P D C 5^{10}$ and a deletion of chromosome 16p11.2. ${ }^{11}$

As PKD is a paroxysmal disorder, it can be misdiagnosed as a seizure disorder, especially when the EEG reveals abnormalities. The patients, then, may be treated as epilepsy with various ASMs, usually at a higher dose than what is used for patients with $P K D^{8}$, as was evident with our patient no. 2. When the diagnosis of PKD was confirmed with a PRRT2 mutation, a low-dose CBZ was given and the paroxysms were completely controlled. An abnormal EEG has been reported in a similar case in a Korean study. ${ }^{17}$ This kind of case emphasizes the need to review the patient's medical history thoroughly, along with the benefit of genetic testing, in order to make a correct diagnosis and provide the appropriate treatment.

This study had some limitations. We enrolled a small number of PKD cases, especially involving familial PKD. This may have affected the detection rate of PRRT2 mutations in our study. Furthermore, we performed only PRRT2 gene sequencing. Therefore, further investigations, such as whole exome sequencing, might help to identify other causative genes. Another point is that our familial cases were only defined by history taking without family genetic testing. Accordingly, it was difficult to show the inheritance pattern. Further genetic testing in families needs to be done.

\section{Conclusion}

Our study provided the new details of the clinical phenotypes and PRRT2 gene analysis findings for Thai PKD. The ratio we identified of PRRT2 mutations corresponds with that in other Asian studies while the familial PKD had a higher detection rate regarding $P R R T 2$ mutations. The finding of c.649dupC (p.Arg217Profs ${ }^{\star} 8$ ) as a hot-spot mutation suggests the priority of detecting c.649dupC in Thai patients with PKD. Although negative genetic findings cannot exclude a diagnosis of PKD, the detection of a PPRT2 mutation could help physicians to make a correct diagnosis, especially when the clinical presentation mimics epileptic seizures. Importantly, a thorough medical history in patients with dyskinesia triggered by initiation of movement is the key to distinguish PKD from epilepsy, which leads to proper management.

\section{Conflict of interest}

None of the authors has any conflict of interest to declare.

\section{Acknowledgement}

The authors are grateful to all the patients and their parents who participated in this study. 


\section{References}

1. Garone G, Capuano A, Travaglini L, Graziola F, Stregapede F, Zanni G, et al. Clinical and genetic overview of paroxysmal movement disorders and episodic ataxias. Int J Mol Sci 2020; 21:3603.

2. Meneret A, Roze E. Paroxysmal movement disorders: an update. Rev Neurol (Paris) 2016;172:433-45.

3. Huang XJ, Wang T, Wang JL, Liu XL, Che XQ, Li J, et al. Paroxysmal kinesigenic dyskinesia: clinical and genetic analyses of 110 patients. Neurology 2015;85:1546-53.

4. Meneret A, Grabli D, Depienne C, Gaudebout C, Picard F, Durr $\mathrm{A}$, et al. PRRT2 mutations: a major cause of paroxysmal kinesigenic dyskinesia in the European population. Neurology 2012;79:170-4.

5. McGuire S, Chanchani S, Khurana DS. Paroxysmal Dyskinesias. Semin Pediatr Neurol 2018;25:75-81.

6. Bruno MK, Hallett M, Gwinn-Hardy K, Sorensen B, Considine E, Tucker $S$, et al. Clinical evaluation of idiopathic paroxysmal kinesigenic dyskinesia: new diagnostic criteria. Neurology 2004; 63:2280-7.

7. Kang SM, Lee JH, Cho KL, Lee HJ, Kim JE, Seo EJ. Clinical manifestation and PRRT2 analysis of Korean patients with paroxysmal kinesigenic dyskinesia. J Korean Child Neurol Soc 2017;25:221-6.

8. Ebrahimi-Fakhari D, Saffari A, Westenberger A, Klein C. The evolving spectrum of PRRT2-associated paroxysmal diseases. Brain 2015;138:3476-95.

9. Gardella E, Becker F, Moller RS, Schubert J, Lemke JR, Larsen $\mathrm{LH}$, et al. Benign infantile seizures and paroxysmal dyskinesia caused by an SCN8A mutation. Ann Neurol 2016; 79:428-36.

10. Cao L, Huang X, Wang N, Wu Z, Zhang C, Gu W, et al.
Recommendations for the diagnosis and treatment of paroxysmal kinesigenic dyskinesia: an expert consensus in China. Transl Neurodegener 2021;10:7.

11. Vlaskamp DRM, Callenbach PMC, Rump P, Giannini LAA, Brilstra EH, Dijkhuizen T, et al. PRRT2-related phenotypes in patients with a 16p11.2 deletion. Eur J Med Genet 2019;62: 265-9.

12. den Dunnen JT, Dalgleish R, Maglott DR, Hart RK, Greenblatt MS, McGowan-Jordan J, et al. HGVS recommendations for the description of sequence variants: 2016 update. Hum Mutat 2016;37:564-9.

13. Richards S, Aziz N, Bale S, Bick D, Das S, Gastier-Foster J, et al. Standards and guidelines for the interpretation of sequence variants: a joint consensus recommendation of the American College of Medical Genetics and Genomics and the Association for Molecular Pathology. Genet Med 2015; 17:405-24.

14. Zhao G, Liu X, Zhang Q, Wang K. PRRT2 mutations in a cohort of Chinese families with paroxysmal kinesigenic dyskinesia and genotype-phenotype correlation reanalysis in literatures. Int J Neurosci 2018;128:751-60.

15. Cao L, Huang XJ, Zheng L, Xiao Q, Wang XJ, Chen SD. Identification of a novel PRRT2 mutation in patients with paroxysmal kinesigenic dyskinesias and c.649dupC as a mutation hot-spot. Parkinsonism Relat Disord 2012;18:704-6.

16. Li HF, Ni W, Xiong ZQ, Xu J, Wu ZY. PRRT2 c.649dupC mutation derived from de novo in paroxysmal kinesigenic dyskinesia. CNS Neurosci Ther 2013;19:61-5.

17. Seo SY, You SJ. Paroxysmal kinesigenic dyskinesia in a patient with a PRRT2 mutation and centrotemporal spike discharges on electroencephalogram: case report of a 10year-old girl. Korean J Pediatr 2016;59:S157-60. 